\title{
Smart Factory im Werkzeugbau bei KAMAX
}

\author{
Reduzierung der Durchlaufzeit um $90 \%$
}

Christian Ludwig, Thomas Farrenkopf, Thomas Panske und Hilmar Gensert, KAMAX Tools \& Equipment GmbH \& Co. KG, Homberg (Ohm)

\begin{abstract}
Unter "Smart Factory" wird die Vision einer Produktionsumgebung verstanden, in der sich Fertigungsanlagen und Logistiksysteme ohne menschliche Eingriffe weitestgehend selbst organisieren [1]. Im Beitrag wird ein Projekt beschrieben, zu dessen Start keiner der Beteiligten das Thema "Smart Factory“ oder „Industrie 4.0" auch nur ansatzweise mit dem Projekt in Verbindung brachte. Vielmehr wurde die Zielsetzung verfolgt, die heutige Lieferzeit von 6-8 Wochen drastisch zu reduzieren. Das Ergebnis ist ein vollständig digitalisierter Geschäftsprozess von der Auftragserstellung, der Produktentwicklung, der Konstruktion, der Fertigung sowie der Abwicklung für "Losgröße 1" mit einer Reduzierung der Durchlaufzeit auf weniger als $10 \%$.
\end{abstract}

Kundenindividuelle Produkte in "Losgröße 1" und "just-in-Time" liefern zu können, sind zwei entscheidende Faktoren, um in Zukunft erfolgreich am Markt zu bestehen. Ein Schlüssel hierzu liegt insbesondere in der Umformtechnik bei den Werkzeugen. Deren Lieferzeit entscheidet oftmals über den Erfolg eines Produkts, insbesondere bei Neuanläufen. Erschwerend kommt hinzu, dass die Lösungsfindung, also die werkzeugseitige Realisierung einer möglichst optimalen Umformstrategie, häufig iterativ verläuft. Die Lieferzeit von Werkzeugen ist dann umso bedeutender. Neben einer ohnehin geforderten hohen Qualität des Produktes und der kosteneffizienten Produktion, steht eine Reduzierung der Lieferzeiten von Werkzeugen damit bei der Herstellung des Kernproduktes besonders im Fokus. Im Fall von KAMAX sind das Verbindungselemente und Bolzen für die Automobilindustrie.

Am Markt sind heute Lieferzeiten von 6-8 Wochen für Umformwerkzeuge üblich. Durch die Individualität der Werkzeuge sind meist handbediente Universalmaschinen im Einsatz. Die aufwändige Logistik lässt keine größeren Strukturen zu, Unternehmen sind meist kleiner als 50 Mitarbeiter, die durchaus mehrere hundert Kunden bedienen.

Die Reduzierung der Lieferzeit um 90\% verlangt eine ganzheitliche Betrachtung des
Geschäftsprozesses und des Produktes. Zunächst wurde das Produkt auf seine Standardisierbarkeit betrachtet. Ausgehend von einem einheitlichen Werkzeugstandard wurde der modulare Werkzeugaufbau umgesetzt. Aufbauend darauf konnten die Produktionsprozesse automatisiert sowie die durchgängige Digitalisierung der Geschäftsprozesse umgesetzt werden. Trotz der hier chronologisch beschriebenen Abfolge sind alle Stufen von Beginn an zu berücksichtigen, da sie in Abhängigkeit stehen und sich wechselseitig beeinflussen. Das "zeitweise" Festlegen von Randbedingungen ist notwendig, bedarf aber der mehrfachen iterativen Anpassung mit steigendem Detaillierungsgrad und Wissenszuwachs.

\section{Standardisieren - Modularisieren - Automatisieren}

Eine Grundbedingung für eine Automatisierung ist die Standardisierung und Modularisierung. Im Projekt wurde das Ziel verfolgt, mindestens $80 \%$ der Warengruppe damit abdecken zu können.

Im vorliegenden Beispiel sind das Einzelwerkzeuge und Matrizen eines Werkzeugsatzes (Bild 1). Für den Fall, dass es sich um gewach-
Dr.-Ing. Christian Ludwig ist Direktor der Abteilung Global Equipment Development bei KAMAXTools \& Equipment und verantwortlich für die Entwicklung von Maschinen \& Verfahren.

Dr. Thomas Farrenkopf ist Direktor der Abteilung Software Engineering bei KAMAX Tools \& Equipment un verantwortlich für das Systemkonzept des vollständig automatisierten End-to-End-Prozesses. Neben seiner Tätigkeit bei KAMAX ist er Lehrbeauftragter an der Technischen Hochschule Mittelhessen (THM).

Thomas Panske ist Entwicklungsleiter der Steuerungstechnik bei KAMAX Tools \& Equipment und verantwortlich für die LMS Systeme sowie deren Schnittstellen zu Bearbeitungs- und Handlingssystemen.

Hilmar Gensert ist CEO von KAMAX Tools \& Equipment.

Hilmar.Gensert@kamax.com www.kamax.com 
Bild 1: Werkzeugsatz.
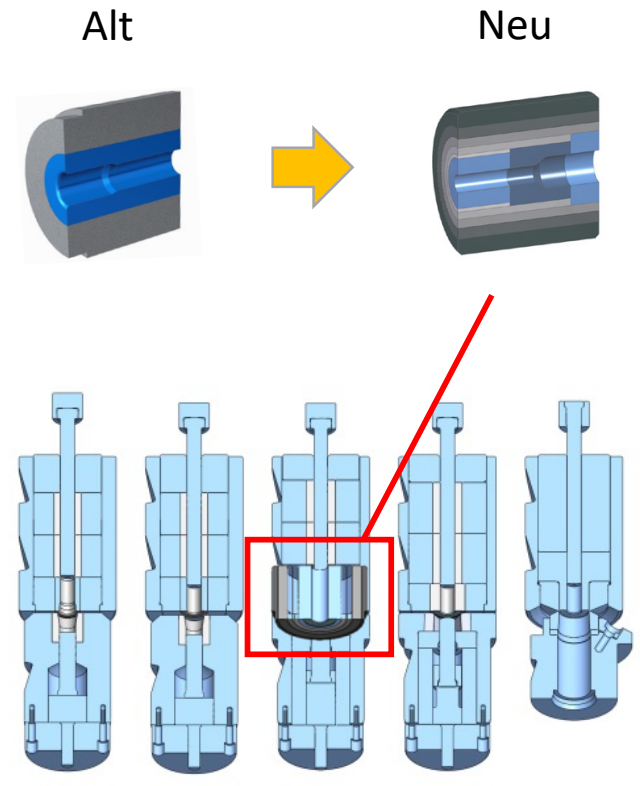

sene Strukturen handelt, ist zudem zu beachten, dass bestehende Schnittstellen zunächst beizubehalten sind. Dies gewährleistet einen stufenweisen Übergang zum neuen Werkzeugkonzept, schränkt aber gleichzeitig die Gestaltungsmöglichkeiten ein.

In Bild 1 wird ein Werkzeugsatz dargestellt, der aus ca. 100 Einzelteilen besteht. Die rot-umrandeten Einzelwerkzeuge, die Matrizen, wurden für das Projekt ausgewählt.

Bei den Matrizen war ein zielführender Ansatz, ein Modulsystem zu entwickeln, aus dem sich alle Fassungsdurchmesser (Außendurchmesser der Matrizen) sowie alle Kerndurchmesser (Hartmetallkerne der Matrizen) abbilden lassen, um mit dem bestehenden System kompatibel zu bleiben. Die Konzeption ist patentrechtlich geschützt.

Aufgebaut aus dem Modulsystem werden die Matrizen jetzt im Durchschnitt fünffach armiert, woraus sich neben der Modularisierung der Matrizenkomponenten auch ein technologischer Vorteil ergibt. So führt die Mehrfacharmierung bis zu einer Verdopplung der Vorspannung des Kerns und somit bei den meisten Einsatzfällen zu einer Vervielfachung der Standmenge.

Um nur mit einer Bearbeitungstechnologie bei der Innenbearbeitung auszukommen, die im Wesentlichen durch das Verhältnis Länge zu Durchmesser der Bohrung bestimmt wird, wurden zudem die Kerne in Abhängigkeit vom Innendurchmesser axial geteilt, $d$. h. die Modularisierung wurde konsequent radial und axial umgesetzt.
Der Schnitt in Bild 1 zeigt einen prinzipiellen Aufbau einer Matrize. Die hohe Anzahl an Einzelkomponenten ist einerseits die modularisierte Lösung, aber andererseits auch eine Herausforderung. Die Schnittstelle zwischen Armierung und Kern ist funktionsbedingt mit toleriert. Das ist bei nur einem Pressverband mit üblicher Fertigungstechnik grundsätzlich herstellbar und ausreichend. Werden beispielsweise acht Ringe ineinandergefügt, entsteht durch die Toleranzkette eine weitere Herausforderung. Eine Erweiterung der Toleranzen ist nicht möglich.

Um dieser Problemstellung zu begegnen, sind iterative Prozesse erforderlich. Die Maschine oder das System hat das erzeugte Bearbeitungsergebnis zu erfassen und die Konsequenzen für die Fertigung der nächsten zur Baugruppe gehörenden Teile abzuleiten. Das heißt, entstehende Fertigungsabweichungen werden jeweils durch nachfolgend gefertigte Baugruppenelemente kompensiert. Ähnlich ist mit der Verschleißkompensation von Werkzeugen zu verfahren. Das Prinzip ist zielführend, geht aber in Abhängigkeit der benötigten Messvorgänge und Iterationsschleifen zu Lasten der benötigten Bearbeitungsdauer. Zur Optimierung der Iterationsschleifen wird ein Predictive Quality Analytics System in Echtzeit genutzt.

\section{Systemlandschaft}

Verfolgt man den Produktentstehungsprozess von der Definition des Produkts über die Fertigung bis zur Anlieferung beim Kunden, ist dieser gekennzeichnet durch eine Vielzahl von Schnittstellen zwischen unterschiedlichen Softwaresystemen. Bilden üblicherweise ERPals auch MES-Systeme seit geraumer Zeit das "IT-Rückgrat" vieler Unternehmen, mangelt es oft an der Durchgängigkeit des Informationsflusses durch alle involvierten Systemen.

Eine wesentliche Komponente in der modernen Systemlandschaft bilden hierbei sowohl die vertikale als auch die horizontale Integration aller beteiligten IT-Systeme und damit deren hierüber abgebildeten Prozesse. Letztere umfassen vor allem auch Maschinen und Anlagen. Neben der kaufmännischen Abwicklung müssen auch sämtliche Engineering-Informationen automatisiert Richtung Produktion transferiert werden.

Nicht selten werden die meisten Schnittstellen manuell bedient. Im einfachsten Fall werden an den Schnittstellen Daten von einem System zum anderen übertragen und ggf. 
ergänzt. Durch eine geschickte Wahl der Systeme mit standardisierten Schnittstellen, kann dies überwunden werden. Um beispielsweise den durchgängigen Datenaustausch und höchsten Automatisierungsgrad für $C A D$, CAM und PDM zu erhalten, kann es nicht nur hilfreich, sondern teilweise auch notwendig werden, neue Systeme in Betracht zu ziehen. Dies spart den Umweg, mit verschiedenen Compilern arbei-

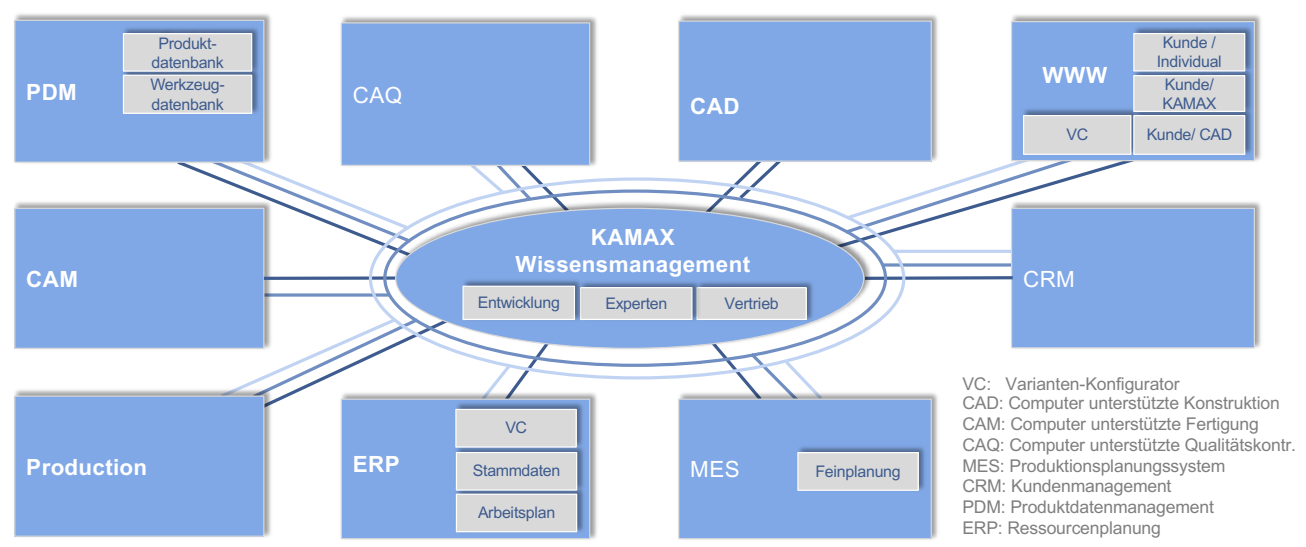
ten zu müssen. In der Regel ist dies nicht nur aufwendiger, sondern schränkt auch die Funktionalität stark ein. Ziel muss es sein, direkt mit nativen Daten zu arbeiten.

Eine weitere Herausforderung stellen Prozesse dar, in denen menschliches Know-how notwendig ist, d. h. es werden Entscheidungen z. B. nach Verfahrensanweisungen gefällt. Solange es dafür ",artikulierbare" Regeln gibt, also externalisierbares Wissen vorliegt, lassen sich diese stark regelbasierten Abläufe meist auch in Algorithmen überführen. Die besondere Herausforderung liegt im impliziten Wissen; Wissen, das auf Erfahrungen, Einstellungen oder Erinnerungen basiert. Neben der ohnehin schon enormen Herausforderung implizites

Wissen in explizites zu verwandeln, stellt sich auch die Frage, wie dieses Wissen und die Erfahrungen maschinell lesbar und verwertbar aufbereitet werden können.

Im vorliegenden Projekt war die Überführung tausender Matrizen in das neue Modul-System erforderlich. Aus diesem Grund wurde ein automatischer Entwurf zwingend erforderlich. Aber entscheidender ist die standardisierte Vorgehensweise, die definitiv nur maschinell sichergestellt werden kann. Dies war die Geburtsstunde für ein „Wissensmanagement-System", welches im Zuge der Entwicklung schnell zum zentralen System aller beteiligten Systeme (Bild 2) wurde.
Bild 2: Systemlandschaft.

Bild 3: Informationsfluss.

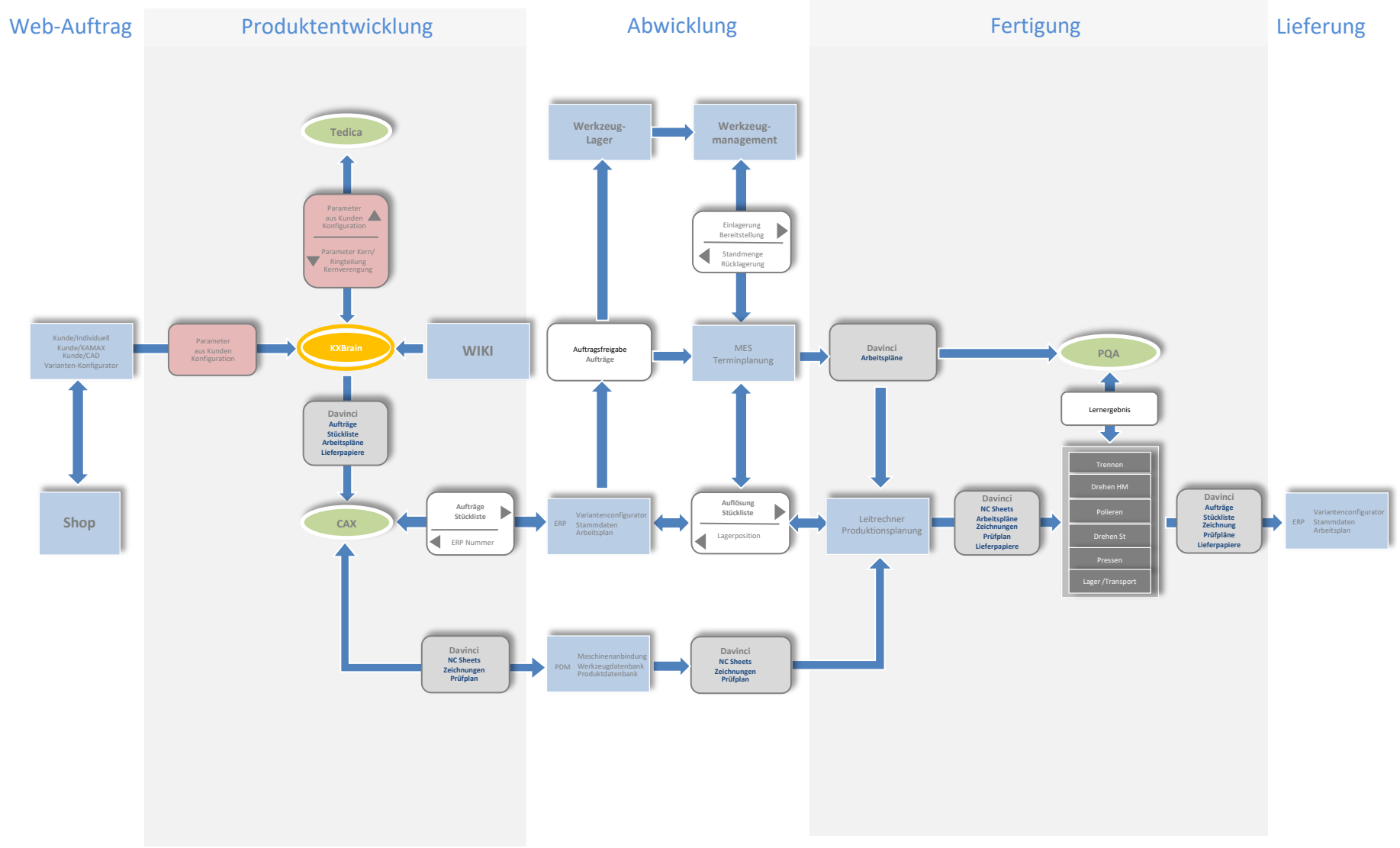




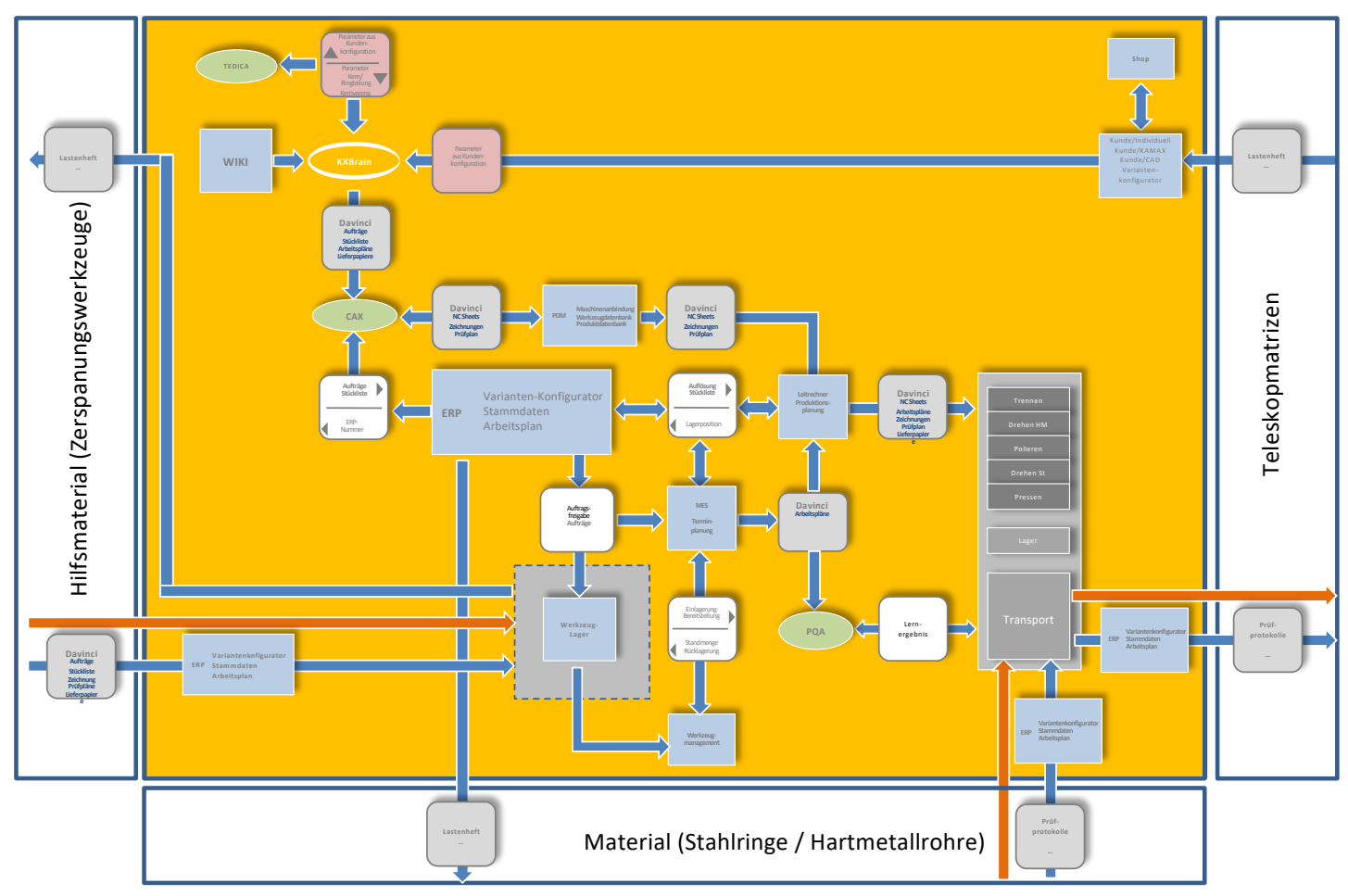

gungsschritt selbst ein. Es werden keinerlei Produktdaten auf den Maschinen abgelegt, da die Fertigung für "Losgröße 1" ausgelegt ist. Das setzt voraus, dass die Maschinen die erforderliche Schnittstelle zur Verfügung stellen.

Komplexer wurde es bei der automatischen Montagezelle. Es wird die Regel sein, dass sich die aufeinander folgenden Werkstücke in den Abmessungen und der Anzahl der zu montierenden Bauteile völlig unterscheiden. Hieraus ergibt sich die Fragestellung, welche Information die Zelle erhal-

Bild 4: Systemgrenzen des Werkzeugbaus.
Relevantes Wissen wird zentral und digitalisiert für alle Systeme maschinenlesbar abgelegt.

Ontologien [2] spielen hierbei eine zentrale Rolle für die formalisierte Repräsentation von Wissen und Informationen. Mithilfe semantisch angereicherter Beschreibungen, ist zudem eine Interpretation der Daten mit Logik und Schlussfolgerungen (sog. Inferenz) möglich.

Das System ist in der Lage, ausgehend von der Eingabe der funktionsrelevanten Daten in ein Web-Frontend, eine komplette Auslegung und Konstruktion der Matrize durchzuführen.

Durchschnittlich sind ca. 10 Eingabevariablen ausreichend, um daraus ca. 800 Variablen zu steuern.

Die Daten werden dann an die weiteren Softwaresysteme wie ERP, PDM, CAD-CAM, MES bis an die Steuerung der Maschinen verteilt. Rückmeldungen zum Auftragsstatus können über das Web-Frontend abgerufen werden. Durch den gewählten Ansatz der Integration von Systemen in einer dynamisch serviceorientierten Umgebung (Enterprise Service Bus) ist es mit dem Wissensmanagement möglich, sowohl die kaufmännischen Prozesse im ERP als auch die technologischen Systeme mit den relevanten Daten zu versorgen, anzutriggern und wiederum erzeugte Daten weiterzuleiten.

Alle Maschinen erhalten demnach bei jedem Teil neue Daten und leiten den nächsten Ferti- ten muss, d. h. wie wird codiert und decodiert?

Um diese Herausforderung zu lösen, wurde ein Code entwickelt, der das Modell und die Eigenschaften beschreibt, der "Digitale Zwilling". Dieser Code wird praktisch mit dem Produkt mitgeführt und steuert alle Bearbeitungs- und Transportfunktionen.

Die Automation birgt aber noch ganz andere Herausforderungen im Informations- und Datenaustausch mit sich. Besonders resistent für derartige Prozesse war das PDM- als auch das ERP-System, da sie statisch arbeiten, aber jetzt echtzeitfähige Bedingungs-Ereignis-Systeme erforderlich sind.

Beispielsweise sind im automatischen Prozess Rohlinge zu entnehmen, Halbfertig- oder Fertigprodukte einzulagern. Reaktionszeiten der Systeme müssen in Echtzeit ablaufen. Eine SPS-Funktion hat aber das ERP-System nicht. Auch stellt die Art der Programmierung des Gesamtsystems eine besondere Anforderung dar. Das Bedingungs-Ereignis-System, das die Steuerung übernimmt, ist so zu gestalten, dass es für alle Beteiligten und nicht nur für die SPS-Programmierer verständlich wird. So liegen die Stärken der Softwaresprachen der SPS-Welt signifikant in ihrer hohen Performance und Echtzeitverarbeitung, hinsichtlich Integrationsfähigkeit, Datenvolumen und Versionsverwaltung bestehen jedoch noch deutliche Schwächen gegenüber IT-Hochsprachen, wie sie in modernen IT-Systemen zum Einsatz kommen. 
Im Zuge der Entwicklungstätigkeiten wurde die Zielsetzung erreicht, die Durchlaufzeit von sechs Wochen auf wenige Stunden zu reduzieren. Zudem haben die modularisierten Werkzeuge ein Vielfaches an Gebrauchswert.

Die Fertigung der "Losgröße 1" nach Kundenvorgabe ist möglich, die Maschinen arbeiten iterativ und das System ist selbstoptimierend. Es wurde eine Produktionsumgebung geschaffen, in der sich Fertigungsanlagen und Logistiksysteme

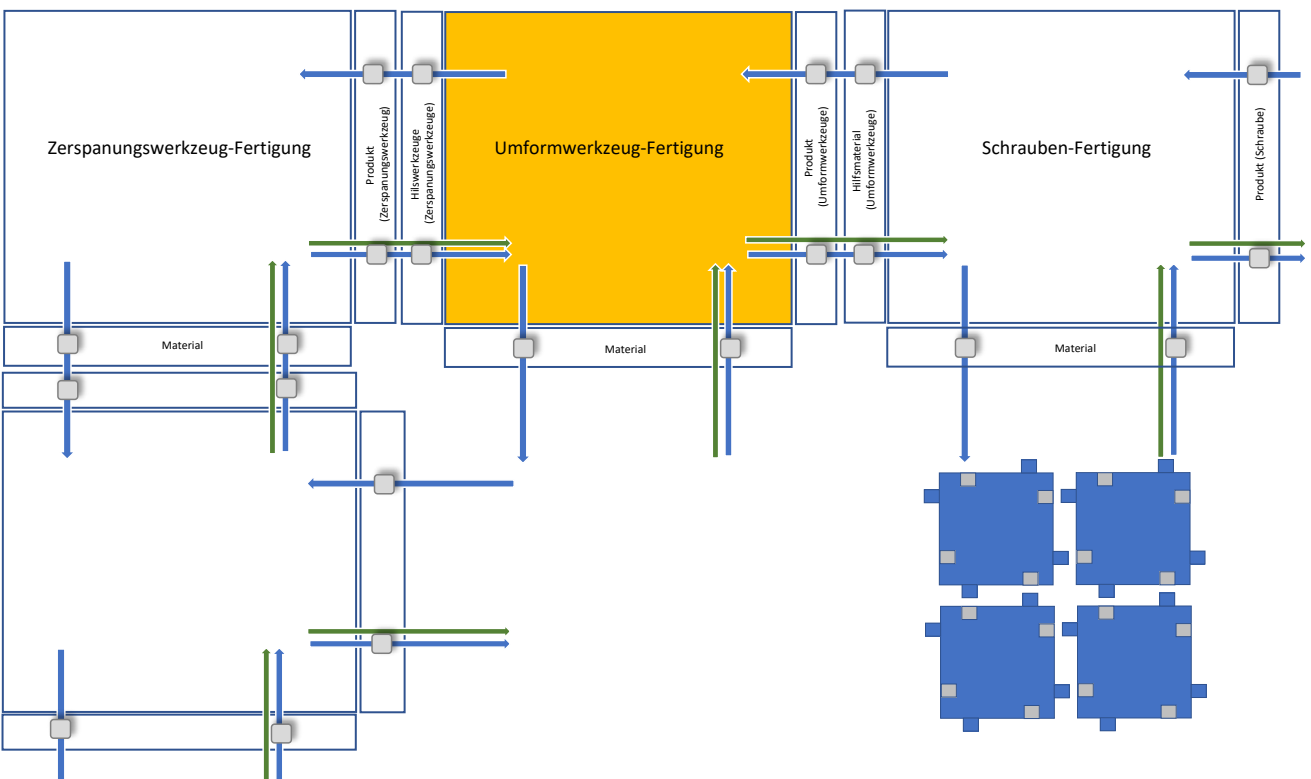
weitgehend selbst organisieren. Alles Eigenschaften, die sich mit dem Thema "Industrie 4.0" oder "Smart Factory" verbinden lassen.

zeugbaus, sondern der gesamten Lieferkette abbildet.

Der finale Prozessablauf des Gesamtsystems wird in Bild 3 gezeigt.

Der Kunde definiert im Shop sein Produkt, erhält sofort einen Preis und kann bestellen (Auftrag). Danach wird, gesteuert durch das Wissensmanagement, das Produkt entwickelt (Produktentwicklung), gefertigt (Fertigung) und geliefert (Abwicklung). Der Kunde erhält nach Auftragserteilung eine Auftragsbestätigung sowie den finalen Liefertermin. Vom Auftrag bis zur Lieferung ist in dem Prozess keine Entscheidung von Mitarbeitern mehr erforderlich.

Das System wurde 2018 durch die Fachzeitschrift Produktion und ROI Management Consulting bewertet und mit dem Industrie 4.0 Award ausgezeichnet $[3,4]$.

\section{Ausblick}

In Bild 3 wurde der Informationsfluss von der Anfrage bis zur Lieferung von Werkzeugen gezeigt. Stellt man das Bild so um, dass die Schnittstellen zu Unterlieferanten und Kunden besser zu erkennen sind, ergibt sich Bild 4. Der Informationsfluss wird durch blaue Linien, der Warenfluss durch orangefarbene Linien symbolisiert.

Aus der Sicht des Kunden (Schraubenfertigung) erscheint der Werkzeugbau als Unterlieferant von Verbrauchsmaterial (Umformwerkzeuge). Damit ergibt sich eine gewisse Modularität, die nicht nur den Geschäftsprozess des Werk-
Bild 4 zeigt die gesamte Lieferkette, wobei das bestehende System nun als Blaupause zur Verfügung steht und durch Modularität sukzessive übertragen wird (Bild 5).

Die Herausforderung besteht darin, das komplexe System servicefreundlich zu machen.

Auch wenn das System robust funktioniert, bleibt es nicht aus, das Störungen auftreten können. In diesem Fall ist ein Experte für das betroffene System notwendig. Auch hier sollen Automatismen realisiert werden, die regelungstechnische Antworten auf Störungen generieren. Die Notwendigkeit der Unterstützung durch Experten soll so sukzessive reduziert werden. Hierfür ist ein selbstlernendes Fehlermanagementsystem in Entwicklung.

Es ist das Ziel, dass bei erstmaligen Auftreten der Störung zunächst weiterhin der Experte diese behebt und der Prozess im Wissensmanagement gespeichert wird. Tritt die Störung ein weiteres Mal auf, wird dem Experten der Ablauf vorgeschlagen, bis der Prozess letztlich vom Wissensmanagement übernommen wird und der Anlagenführer nur noch den Anweisungen zu folgen hat.

Der vorliegende Beitrag wurde in abgewandelter Form bereits in [5-7] veröffentlicht.

\section{Schlüsselwörter:}

Smart Factory, Smart Supply Chain, Industrie 4.0, Automatisierung, Wissensmanagement, Autonome Produktionssteuerung, Losgröße 1
Bild 5: Smart Supply Chain.

Literatur

[1] Bracht, U.; Geckler, D.; Wenzel, S.: Digitale Fabrik. VDI-Buch Berlin Heidelberg 2011.

[2] Grimm, S.; Hitzler, P.; Abecker, A.: Knowledge Representation and Ontologies. In: Studer, R. Grimm, S.; Abecker, A. (Hrsg): Semantic Web Services. Berlin Heidelberg 2007.

[3] ROI: BMW, SIEMENS UND KAMAX TOOLS \& EQUIPMENT sind Deutschlands Industrie 4.0-Champions. URL: www. roi.de/news/artikel/news/ bmw-siemens-und-kamaxto o I s-equipment- $\mathrm{s}$ i $\mathrm{n}$ d -deutschlands-industrie-40champions, Abrufdatum 01.01.2021

[4] Industrie 4.0 Award: Die Besten der Besten. URL www. industrie40award.de/ smart-scm-2018, Abrufdatum 01.01.2021.

[5] Gensert, H.; Ludwig, C.: Smart factory. Tagungsband 33. VDI Jahrestreffen der Kaltmassivumformer 2018.

[6] Gensert, H.; Ludwig, C.: Smart factory. Tagungsband Umformtechnisches Kolloquium. Darmstadt 2018

[7] Gensert, H.; Ludwig, C.; Farrenkopf, T:; Panske, T.: Smart Factory im Werkzeugbau, Umformtechnik Massiv+Leichtbau. Bamberg 2021. 